\title{
Double Terminal Sliding Mode Control of Airship Height Loop
}

\author{
Yidong Wang ${ }^{1, \mathrm{a}}$, Jinhuang $\mathrm{Wu}^{1}{ }^{1}$, Dingxiong Zhang $^{1}$ \\ Hong Wang ${ }^{2}$ and Junwei Lei ${ }^{2}$ \\ ${ }^{1}$ The $91872^{\text {th }}$ unit of PLA, Beijing, China
}

${ }^{2}$ Department of control engineering, Naval aeronautical and Astronautical University, Yantai, 264001

awangyidong96107@163.com

Keywords: airship, pitch channel, adaptive, PID, stability

\begin{abstract}
A kind of double terminal sliding mode surface is constructed with a subsystem with a terminal sliding mode. This new strategy is proposed to solve the height control problem of a kind of airship system. And the main goal is to compute a ideal pitch angel such that the height of airship can trace the expected value. Also a Lyapunov stability theory is used to prove the whole system is stable. And at last, detailed simulation is done to testify the rightness of this method.
\end{abstract}

\section{Introduction}

As early as more than a century ago, Germany, Russia began to research and produce the airship, although airship technology development after a period of twists and turns, but since 1994, the world began to reconsider the airship for commercial and military value, and airship technology began to attract people's eyes, airship technology began to enter a period of prosperity[1-5]. The airship control technology research mainly concentrated in the inner loop of the system is stable or attitude stabilization, and emerged on top of the basic attitude stabilization height control external circuit research method is not much, most still use PID control method[6-10]. Mainly because the PID control method can satisfy the control task of the slow maneuver in some small airspace. But when the airship is in large scale maneuver and has a certain requirement of rapidity, the conventional PID control method is difficult to meet the requirements. Based on the above reasons, this paper attempts to study a kind of dual sliding mode control strategy Terminal method.

\section{Model Description}

Based on the previous work, the pitch channel model of airship can be described as follows:

$$
M \dot{x}=f(x)+g(x) u
$$

And $x=\left[\begin{array}{llllll}u & w & q & \theta & x & z\end{array}\right], M$ satisfies

$$
M^{-1}=\left[\begin{array}{llllll}
a_{11} & & a_{13} & & & \\
& a_{22} & & & & \\
a_{31} & & a_{33} & & & \\
& & & 1 & & \\
& & & & 1 & \\
& & & & & 1
\end{array}\right]
$$

The definition of $a_{i j}$ see the definition of $M$ in previous work.

Choose the expect value of all states $u, w, q, \theta, x, z$ are $u^{d}, w^{d}, q^{d}, \theta^{d}, x^{d}, z^{d}$, Define the error variable $e=x-x^{d}, \dot{e}=\dot{x}$, then it hold[6-7]

$$
M \dot{e}=f(x)+g(x) u
$$

Use the inverse matrix of $M$ 


$$
\dot{e}=M^{-1} f(x)+M^{-1} g(x) u
$$

To make it convenient for reading, some functions can be written as follows

$$
f(x)=\left[\begin{array}{l}
f_{1} \\
f_{2} \\
f_{3} \\
f_{4} \\
f_{5} \\
f_{6}
\end{array}\right] u=\left[\begin{array}{ll}
u_{1} & u_{2}
\end{array}\right]^{T}
$$

where

$$
\left[\begin{array}{c}
f_{1} \\
f_{2} \\
f_{3} \\
f_{4} \\
f_{5} \\
f_{6}
\end{array}\right]=\left[\begin{array}{c}
-\left(m+m_{33}\right) w q+Q\left[C_{X 1} \cos ^{2} \alpha+C_{X 2} \sin (2 \alpha) \sin (\alpha / 2)\right. \\
\left(m+m_{11}\right) q u+m a_{z} q^{2}+Q\left[C_{z 1} \cos (\alpha / 2) \sin (2 \alpha)+C_{z 2} \sin (2 \alpha)+C_{z 3} \sin (\alpha) \sin (|\alpha|)\right] \\
-m a_{z} w q(-r v)+Q\left[C_{M 1} \cos (\alpha / 2) \sin (2 \alpha)+C_{M 2} \sin (2 \alpha)+C_{M 3} \sin (\alpha) \sin (\mid \alpha)\right]-a_{z} \sin \theta W \\
q \\
u \cos \theta+w \sin \theta \\
-u \sin \theta+w \cos \theta
\end{array}\right]
$$

Define

$$
M^{-1} f(x)=\left[\begin{array}{c}
f_{a 1} \\
f_{a 2} \\
f_{a 3} \\
f_{a 4} \\
f_{a 5} \\
f_{a 6}
\end{array}\right]=\left[\begin{array}{c}
a_{11} f_{1}+a_{13} f_{3} \\
a_{22} f_{2} \\
a_{31} f_{1}+a_{33} f_{3} \\
f_{4} \\
f_{5} \\
f_{6}
\end{array}\right]
$$

And

$$
g(x) u=\left[\begin{array}{c}
u_{2} \\
k_{1} u_{1} \\
k_{2} u_{1} \\
0 \\
0 \\
0
\end{array}\right]
$$

Then the system can be written as follows[8-9]

$$
\left[\begin{array}{c}
\dot{u} \\
\dot{w} \\
\dot{q} \\
\dot{\theta} \\
\dot{x} \\
\dot{z}
\end{array}\right]=\left[\begin{array}{c}
f_{a 1} \\
f_{a 2} \\
f_{a 3} \\
f_{a 4} \\
f_{a 5} \\
f_{a 6}
\end{array}\right]+\left[\begin{array}{c}
a_{11} u_{2}+a_{13} k_{2} u_{1} \\
a_{22} k_{1} u_{1} \\
a_{31} u_{2}+a_{33} k_{2} u_{1} \\
0 \\
0 \\
0
\end{array}\right]
$$

\section{Sliding Mode Controller Design for Height Loop}

Below design and research is based on the assumption that the pitch angle control system of airship is effective so the goal of height loop is to design the desired or expected value of pitch angle.

Assume the expected height is $z^{d}$, and define the error variable as 


$$
e_{z}=z-z^{d}
$$

Then according to the above model, its derivative can be solved as

$$
\dot{e}_{z}=\dot{z}=-u \sin \theta+w \cos \theta
$$

And choose a integral sliding mode surface as

$$
s_{z}=c_{a z} s_{a z}+\left(\int s_{a z} d t\right)^{9 / 13}
$$

Where

$$
S_{a z}=c_{z} e_{z}+\left(\dot{e}_{z}\right)^{11 / 13}
$$

Solve its derivative as

$$
\begin{aligned}
& \dot{s}_{z}=c_{a z} \dot{s}_{a z}+\frac{9}{13}\left(\int s_{a z} d t\right)^{-4 / 13} s_{a z} \\
& =c_{a z} c_{z} \dot{e}_{z}+c_{a z} \frac{11}{13}\left(\dot{e}_{z}\right)^{-2 / 13} \ddot{e}_{z}+\frac{9}{13}\left(\int s_{a z} d t\right)^{-4 / 13} s_{a z} \\
& =-c_{z} c_{a z} u \sin \theta+c_{z} c_{a z} w \cos \theta+c_{a z} \frac{11}{13}\left(\dot{e}_{z}\right)^{-2 / 13}(-u \sin \theta+w \cos \theta)^{\prime}+\frac{9}{13}\left(\int s_{a z} d t\right)^{-4 / 13} s_{a z}
\end{aligned}
$$

where

$$
(-u \sin \theta+w \cos \theta)^{\prime}=-\dot{u} \sin \theta+\dot{w} \cos \theta-(u \cos \theta+w \sin \theta) q
$$

then

$$
\begin{aligned}
& \dot{s}_{z}=-c_{z} c_{a z} u \sin \theta+c_{z} c_{a z} w \cos \theta+\frac{9}{13}\left(\int s_{a z} d t\right)^{-4 / 13} s_{a z} \\
& +c_{a z} \frac{11}{13}\left(\dot{e}_{z}\right)^{-2 / 13}[-\dot{u} \sin \theta+\dot{w} \cos \theta-(u \cos \theta+w \sin \theta) q]
\end{aligned}
$$

Design the expected value of pitch angle as

$$
\theta=\left[k_{z 1} s_{z}+k_{z 2} s_{z} /\left(\left|s_{z}\right|+\xi\right)\right]+d_{t} \operatorname{sign}\left(s_{z}\right)
$$

And define

$$
\begin{aligned}
& \Delta=c_{z} c_{a z} w \cos \theta+\frac{9}{13}\left(\int s_{a z} d t\right)^{-4 / 13} s_{a z} \\
& +c_{a z} \frac{11}{13}\left(\dot{e}_{z}\right)^{-2 / 13}[-\dot{u} \sin \theta+\dot{w} \cos \theta-(u \cos \theta+w \sin \theta) q]
\end{aligned}
$$

where

$$
\dot{s}_{z}=-c_{z} c_{a z} u \sin \left[k_{z 1} s_{z}+k_{z 2} s_{z} /\left(\left|s_{z}\right|+\xi\right)\right]+\Delta
$$

For the following inequality, there exist positive constant $k_{p 1}$ and $k_{p 2}$ such that

$$
k_{p 1} \theta^{2} \leq \theta \sin \theta \leq k_{p 2} \theta^{2}
$$

For a small $\theta$, when $\Delta$ compare with $\theta$, it can satisfy $\Delta<d_{r} \theta$, so

$$
\theta \dot{s}_{z}=-c_{z} c_{a z} \theta u \sin [\theta]+\Delta \theta
$$

Then it holds

$$
\left[k_{z 1}+k_{z 2} /\left(\left|s_{z}\right|+\xi\right)\right] s_{z} \dot{s}_{z} \leq-c_{z} c_{a z} u k_{p 1} \theta^{2}+d_{r} \theta^{2}
$$

(21)

So it is easy to choose proper control gain such that

$S_{z} \dot{S}_{z} \leq 0$

And according to Lyapunov stable theorem, the system is stable. 


\section{Numerical Simulation}

To testify the rightness of the above method, a numerical simulation is done as follows: choose control parameter as $c_{z}=0.06$, set $k_{z 1}=0.1, k_{z 2}=5, \xi=10$, then assume $u=20 \mathrm{~m} / \mathrm{s}, T_{m}=8000 \mathrm{~N}$, initial position $x_{e}=y_{e}=z_{e}=0$. Other quantity of state is 0 , choose $z_{d}=-500$, simulation results are as following figures:

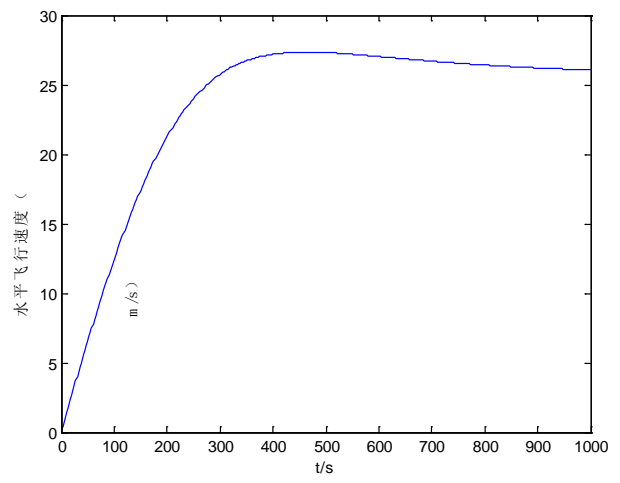

Fig.2. Forward Velocity

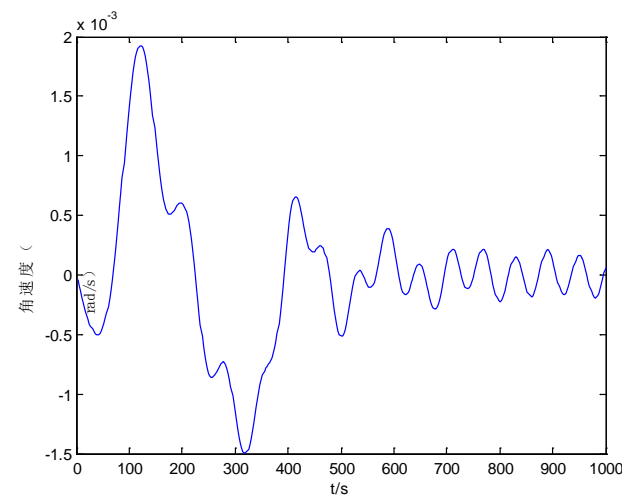

Fig. 4. Angle Velocity

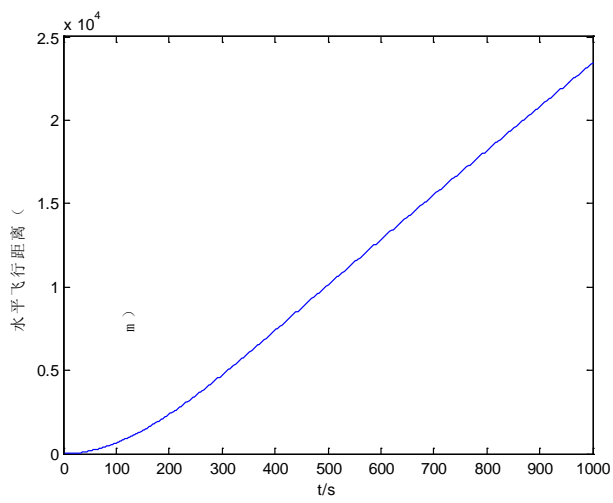

Fig. 6. Flying Distance

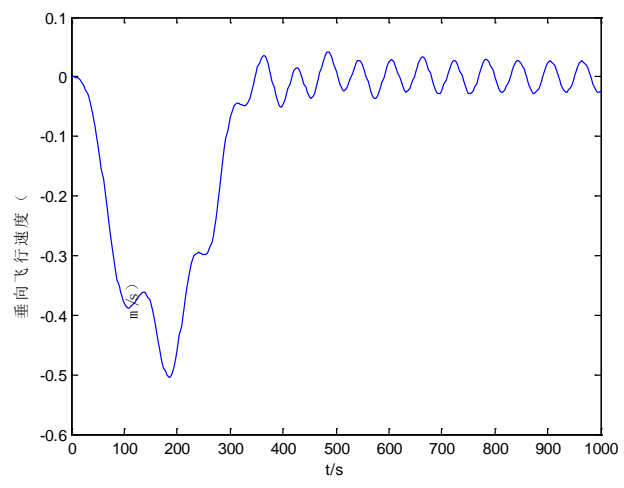

Fig.3. Vertical Velocity

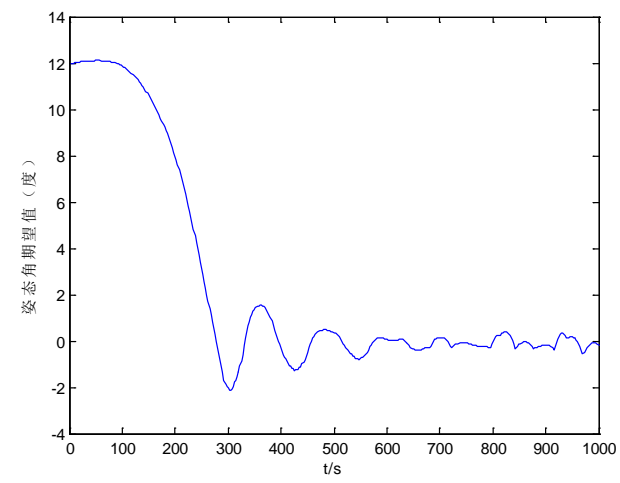

Fig.5. Pitch Angle

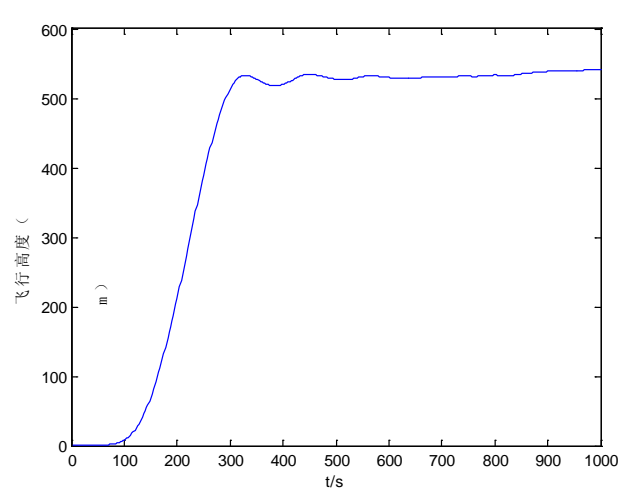

Fig. 7. Height 


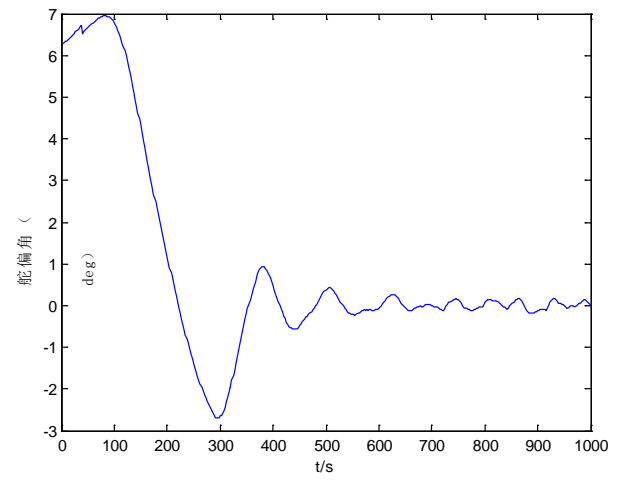

Fig. 8. Pitch Actuator Angle

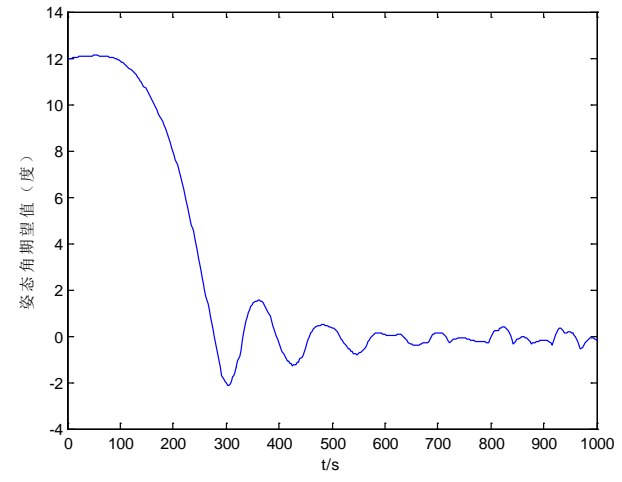

Fig. 9. Expected value of pitch angle

The simulation results show that airship can follow the tracks of expected height $500 \mathrm{~m}$ but there exists overshoot and steady state error, and the pitch angle and actuator angle also exists chattering problem. It means that the system does not has enough damping ratio, and this is because there is no derivative signal in the whole control law.

\section{Conclusion}

A kind of novel sliding mode method is used to solve the height tracing problem of airship system, where sliding mode surface is constructed with a big terminal surface, and the big terminal surface is composed with a small terminal surface. The whole strategy which is proved to be stable by Lyapunov stability theorem, is used to generate a ideal pitch angle of airship system. And the simulation result shows that the proposed method is right but the dynamic performance is not very good because of the appearance of steady state error and oscillations. This disadvantage can be solved by introducing damping ratio or properly setting of terminal sliding mode parameters, which will be the main point of our future research.

\section{References}

[1] J.S.Uhlman, N.E.Fine, D.C.Kring. Calculation of the Added Mass and Damping Forces on Supercavitating Bodies.The 4th International Symposium on Cavitation,California,2001:7 13

[2] D.Clarke.Calculation of the Added Mass of Elliptical Cylinders in Shallow Water.Ocean Engineering.2001,28(4):61 72

[3] C.J.Atkinson, R.G.Urso. Modeling of Apparent Mass Effects for the Real-Time Simulation of a Hybird Airship.AIAA Modeling and Simulation Technologies Conference and Exhibit,Keystone.2006:21 32

[4] Yokomaku Y.The Stratospheric Platform Airship R\&D Program of Japan. The 2nd Stratospheric Platform Systems Workshop, Tokyo Japan.2000:7 13

[5] S.P.Jones,J.D.Laurier.Aerodynamic Estimation Techniques for Aerostats and Airships.AIAA Lighter-than-Air Systems Conference,Annapolis,2004: 88 94

[6] M.T.Soylemeza, N.Munrob, H.Bakic. Fast Calculation of Stabilizing PID Controllers.Automatic,2003,39(7):121 126

[7] Etkin B, Theory of the flight of Airplanes in Isotropic Turbulence Review an Extension, AGARD Rept.1961:372

[8] David K. Schmidt, James Stevens, Jason Roney. Dynamic Modeling ,Control, and Station-Keeping Guidance of A Large High-Altitude "Near-Space" Airship. AIAA Guidance, Navigation, and Control Conference and Exhibit. 2006-6781

[9] David K. Schmidt. Modeling and Near-Space Station-Keeping Control of a Large High-Altitude Airship. Journal of Control and Dynamics,2007,30(2):540 547

[10] Azinheira, J.R., A.Moutinho, Hover Control of an UAV With Backstepping Design Including Input Saturations[J]. IEEE Transactions on Control System Technology, 2008.16(3):517-526. 